\title{
Sintesi delle proposte emerse dalla prova pratica relativa al Corso di Aggiornamento tenuto dall'Amcli - Cotelab a Rovereto il 23-24 settembre 2003 e a Pordenone 2-9 ottobre 2003
}

\section{ROVERETO}

a) Definire il concetto di "NON CONFORMITÀ"

b) Progettare una scheda per la rivelazione delle non conformità.

I partecipanti sono stati suddivisi in 4 gruppi di lavoro

\section{Risultati \\ NON CONFORMITÀ}

Gruppo A: "Si definisce materiale non conforme quello non adeguato all'esecuzione dell'indagine stessa"

\section{ESERCITAZIONE PRATICA:}

Gruppo B: "Il campione non conforme è:

a) non adeguato al tipo di esame richiesto

b) non rispetta le caratteristiche degli standard di sicurezza

c) quello che per qualche peculiarità non permette il completo svolgimento dell'iter diagnostico"

Gruppo C: La non conformità è l'inosservanza delle linee guida definite

Gruppo D: "La non conformità è l'insieme degli elementi, fattori che possono influenzare negativamente il risultato e/o inficiare la qualità del processo."

PROPOSTA DI SCHEDA DI RILEVAZIONE DELLE NON CONFORMITÀ Gruppo A:

\begin{tabular}{|c|c|c|c|c|}
\hline DATA & PAZIENTE & REP & $\begin{array}{l}\text { TIPO DI NON } \\
\text { CONFORMITÀ }\end{array}$ & $\begin{array}{l}\text { REFERENTE } \\
\text { LABORATORIO }\end{array}$ \\
\hline & & & PRELIEVO .INSUFFICIENTE & \\
\hline & & & CONTENITORE NON IDONEO & \\
\hline & & & CAMPIONE ANONIMO & \\
\hline & & & $\begin{array}{l}\text { RICHIESTA INCOMPLETA O } \\
\text { NON PERVENUTA }\end{array}$ & \\
\hline & & & CAMPIONE NON PERVENUTO & \\
\hline & & & $\begin{array}{l}\text { CAMPIONE PERVENUTO } \\
\text { FUORI ORARIO }\end{array}$ & \\
\hline & & & $\begin{array}{l}\text { CONSERVAZIONE NON } \\
\text { CORRETTA }\end{array}$ & \\
\hline & & & $\begin{array}{l}\text { MANCANZA DI TERRENO DI } \\
\text { TRASPORTO }\end{array}$ & \\
\hline & & & ALTRO & \\
\hline
\end{tabular}

\section{Gruppo B:}

\section{TIPO DI NON CONFORMITÀ:}

a) Contenitori e campioni non idonei al tipo di richiesta

b) Inadeguato rispetto dei tempi di trasporto e di conservazione

c) Contenitore aperto,imbrattato,non identificato

d) Non corretta compilazione della richiesta di accompagnamento (scheda o impegnativa)

e) Mancanza di anamnesi clinica del paziente
Gruppo C:

TIPO DI NON CONFORMITÀ:

Richiesta:

a) incompleta

b) assente

c) sporca

Campione:

a) insufficiente

b) non idoneo

c) anonimo

Raccolta

b) contenitore non idoneo 
Conservazione: a) tempi di consegna non Gruppo D: rispettati

b) temperatura non rispettata

\section{TIPO DI NON CONFORMITÀ:}

Trasporto: contenitori secondari inadeguati
a) Contenitori non idonei
b) Quantità insufficiente del campione
c) Errata identificazione del paziente
d) Contenitore contaminato o non conforme alle norme di sicurezza
e) Errata accettazione del campione

\section{PORDENONE}

\section{PROPOSTA DI SCHEDA DI RILEVAZIONE DELLE NON CONFORMITÀ}

\section{Gruppo A:}

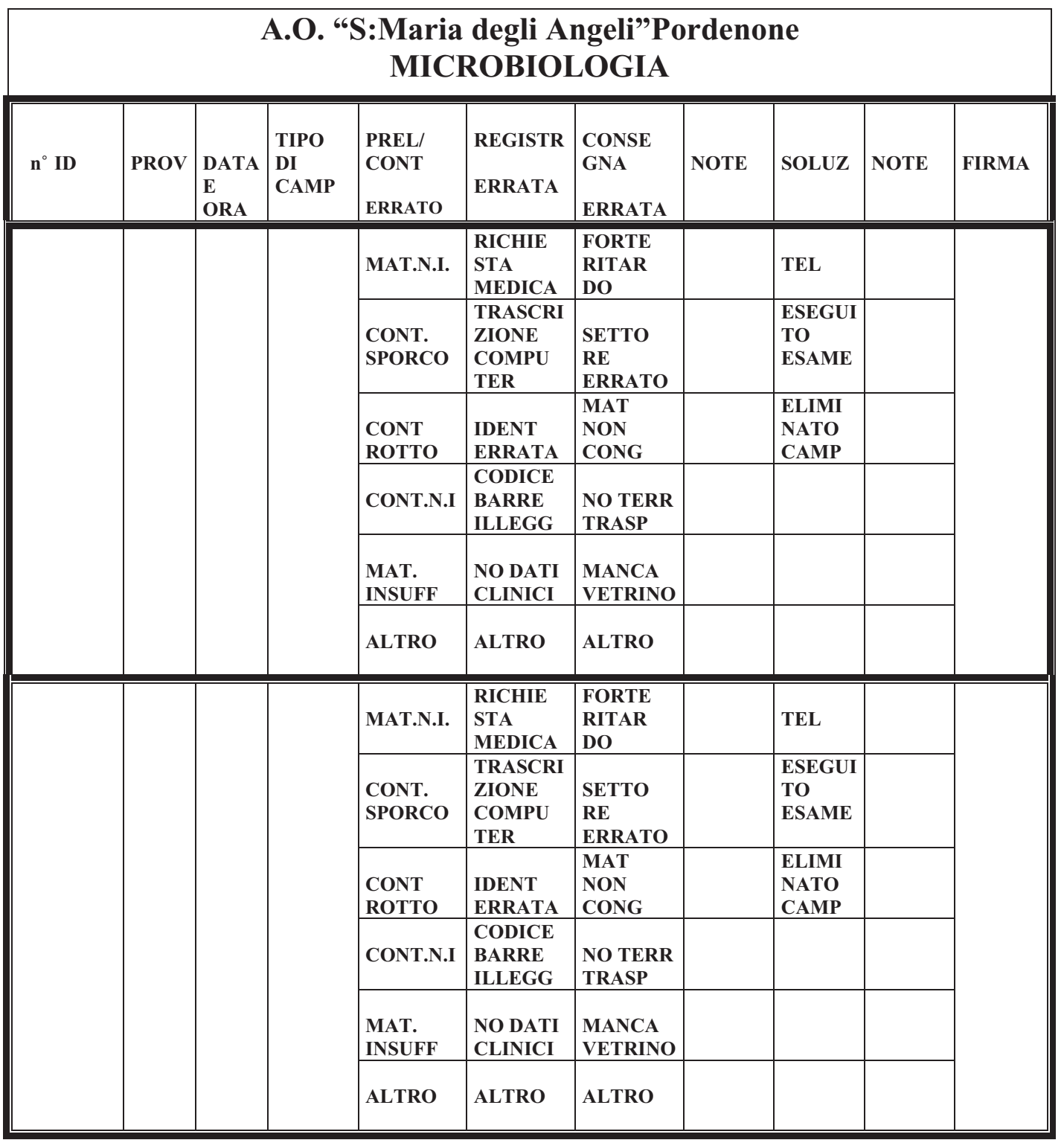




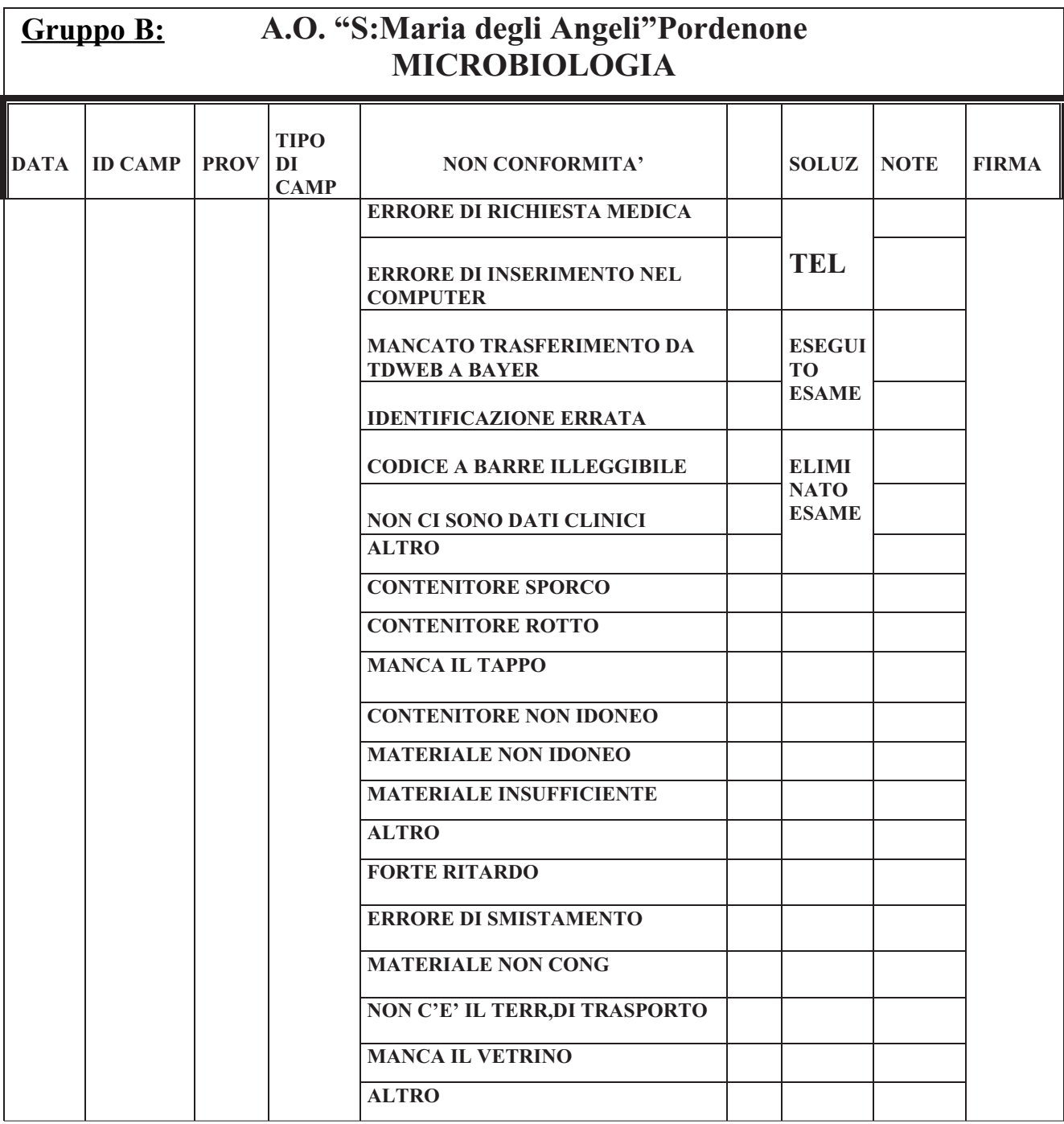

Gruppo D: $\quad$ A.O. "S:Maria degli Angeli"Pordenone

\begin{tabular}{|l|l|l|l|l}
\hline MICROBIOLOGIA & DATA & FIRMA & PROV & PAZIENTE \\
\hline \begin{tabular}{|l|l|l}
\hline NON CONFORMITA' \\
RILEVATA
\end{tabular} & & & \\
\hline RICHIESTA MANCANTE & & \\
\hline RICHIESTA INCONGRUA & & \\
\hline RICHIESTA INCOMPLETA & & \\
\hline CAMPIONE NON & & \\
\hline CAMPIONE NON IDONEO & & \\
\hline CAMPIONE CONTAMINATO & & \\
\hline SOLUZIONE PROBLEMA & & \\
\hline COMUNICATO A: & & \\
\hline PROCESSATO IL CAMPIONE & & \\
\hline ELIMINATO IL CAMPIONE & & \\
\hline
\end{tabular}


PROPOSTA DI SCHEDA PER IL RILEVAMENTO DELLE "NON CONFORMITÀ" DEL CAMPIONE PER INDAGINI MICROBIOLOGICHE

\begin{tabular}{|c|c|c|c|c|c|c|c|}
\hline \multicolumn{8}{|c|}{$\begin{array}{c}\text { INTESTAZIONE STRUTTURA } \\
\text { MICROBIOLOGIA }\end{array}$} \\
\hline $\mathbf{N}^{\circ}$ & DATA & $\begin{array}{l}\text { ID CAMPIONE } \\
\text { TIPO MATERIALE }\end{array}$ & PROV & $\begin{array}{l}\text { DESCRIZIONE NON } \\
\text { CONFORMITA' } \\
\text { sigla }\end{array}$ & FIRMA & $\begin{array}{l}\text { AZIONE } \\
\text { CORRET- } \\
\text { TIVA } \\
\text { sigla }\end{array}$ & LEGENDA N.C. \\
\hline 1 & & & & & & & \multirow{4}{*}{$\begin{array}{l}\text { DOCUMENTAZIONE } \\
\text { NON CONFORME } \\
\text { A: RICHIESTA } \\
\text { B: IDENT. ERRATA } \\
\text { C: COD. BARRE } \\
\quad \text { ILLEGGIBILE } \\
\text { F: DATI CLINICI } \\
\text { G: MANCA AUTORIZ. } \\
\text { H: ALTRO }\end{array}$} \\
\hline 2 & & & & & & & \\
\hline 3 & & & & & & & \\
\hline 4 & & & & & & & \\
\hline 5 & & & & & & & \multirow{4}{*}{$\begin{array}{l}\text { PRELIEVO NON } \\
\text { CONFORME } \\
\text { 1: CONT, SPORCO } \\
\text { 2: CONT ROTTO } \\
\text { 3: MANCA IL TAPPO } \\
\text { 4:CONT.NON IDONEI } \\
\text { 5:MATER.NON } \\
\text { IDONEO } \\
\text { 6:MATERIALE } \\
\text { INSUFFICIENTE } \\
\text { 7: CAMPIONE NON } \\
\text { PERVENUTO } \\
\text { 8: ALTRO } \\
\end{array}$} \\
\hline 6 & & & & & & & \\
\hline 7 & & & & & & & \\
\hline$\cdots$ & & & & & & & \\
\hline & & & & & & & \multirow{3}{*}{\begin{tabular}{|l|} 
TRASPORTO E \\
CONSERVAZIONE \\
NON CONFORMI \\
\\
FORTE RITARDO \\
ERRORE DI \\
SMISTAMENTO \\
CAMP.NON CONG
\end{tabular}} \\
\hline & & & & & & & \\
\hline & & & & & & & \\
\hline & & & & & & & \multirow{2}{*}{$\begin{array}{l}\text { SOLUZIONI } \\
\text { R: RICHIESTO ALTRO } \\
\text { CAMPIONE } \\
\text { T: TELEFONATO } \\
\text { ES : ESEGUITO } \\
\text { COMUNQUE } \\
\text { EL: ELIMINATO } \\
\text { AL: ALTRO } \\
\end{array}$} \\
\hline & & & & & & & \\
\hline
\end{tabular}

\begin{tabular}{|l|}
\hline $\mathbf{N}^{\circ}$ Altro: \\
\hline $\mathbf{N}^{\circ}$ _ Altro: \\
\hline $\mathbf{N}^{\circ}$ _ Altro:
\end{tabular}

